\title{
New Methods for Defining and Investigating Human Consciousness: 2015 Grandy JK*
}

North Country Urgent Care, Watertown New York, USA

\section{Introduction}

Historically, defining consciousness has been exceedingly difficult and perplexing to neurologists and neuroscientists. Studying consciousness objectively in the human brain has been exponentially problematic. However, as newer technological approaches evolve and more is understood about the complexities of the human brain, novel ways of objectively understanding human consciousness have emerged. In this paper I briefly review two new methods of defining consciousness with the novel EPIC consciousness paradigm and the neurogenetic model; as well as two new neurophysiological discoveries that change the way human consciousness is objectified. Collectively, these breakthroughs may enable investigators to better understand both human consciousness and disorders of consciousness.

\section{A new paradigm for defining consciousness}

A new classification system integrating, what is being referred to as "EPIC prongs", has been established by neuropsychiatrist Dr. Vernon M. Neppe and physicist Dr. Edward R. Close. In their recent paper "EPIC Consciousness: A Pertinent New Unification of an Important Concept" they deduce four factors that contribute to the difficulty of describing consciousness [1]:

1. The everyday use of the concept of "consciousness" has varied.

2. Different specialists conceptualize it from their specific framework.

3. Even within these specific groups, it may be applied variably, imprecisely, and ambiguously.

4. There are many components to consciousness and few recognize that these different elements should be assessed.

Dr. Neppe and Dr. Close maintain that consciousness should be precisely conceptualized. In order to accomplish this goal and to ensure that all the key elements are applied to any specific description of consciousness they relate a new four-pronged approach called the "EPIC classification of consciousness". These EPIC prongs are summarized as:

E: Existential "distinctions" of Consciousness are further subdivided into "extent, content and impact distinctions"

1. Consciousness extent substrate: measurable ordinal Consciousness dimensions are tethered to measurable Space and Time dimensions.

2. Consciousness content matrix: This Consciousness container is comparable with mass- energy containers.

3. Consciousness impact: Consciousness impacts and influences the "extent" and "content".

P: Paradigmatic levels of Consciousness: Consciousness involves a four-level gradation- keep in mind that all four of these levels are applicable to living humans

1. Qualit Consciousness: the most basic consciousness (Qualit) level always exists in everything inanimate or animate as everything contains the most fundamental discrete finite physical meaning. Qualits are quanta plus meaning.
2. Neurobiological/ Neurological Consciousness: the endpoint nervous system expression of all living (animate) beings. They have awareness and responsiveness.

3. Psychological Consciousness: involving humans and animals. The psychological is disputably partly separated from the neurological.

4. Higher Consciousness disputably outside the brain: This might involve dreams, meditation, creative, transcendent, psi and altered states, plus mystical traits.

I: Information converted to meaning: Infinitely large repositories of general information are expressed as direct targeted, specific meaningful information.

C: Cybernetic consciousness communications: This provides a mechanistic input, central and output model, applicable to any consciousness models like stimulus organ-response, dendrite-neuronaxon, or stimulus-brain (central)-motor.

According to Dr. Close and Dr. Neppe, the four EPIC prongs are always applied together, reflecting the unification of consciousness in its broadest general applications.

The establishment of a neurogenetic model of human consciousness: The neurogenetic model of human consciousness is based on the phenomenon of what are referred to as neurogenetic correlates of consciousness ( $\mathrm{NgCC})$. $\mathrm{NgCC}$ are defined as genes and gene-derived products, e.g., RNA and epigenetic factors that underlay three processes [2]:

1. Give rise to regions of the brain that ultimately participate in conscious activity

2. Are involve in the continuum of consciousness throughout the lifespan

3. Are involved in the later phases of neuron degeneration which results in decreases in the degree of consciousness.

Hence there are three neurogenetic phase of human consciousness that have been outlined in previous works $[2,3]$ :

1. The first neurogenetic phase- involves genes that are involved in the emergence of brain regions that are involved in sensory information input, interpretation, computation, cognition, interaction, complexity of response, and memory- to name a few.

*Corresponding author: Dr. John K. Grandy, North Country Urgent Care Watertown New York, USA, Tel: 3157863436; E-mail: khyber_john@yahoo.com

Received February 22, 2015; Accepted May 26, 2015; Published May 29, 2015

Citation: Grandy JK (2015) New Methods for Defining and Investigating Human Consciousness: 2015. J Neurol Disord 3: e114. doi: 10.4172/2329-6895.1000e114

Copyright: ( 2015 Grandy JK. This is an open-access article distributed under the terms of the Creative Commons Attribution License, which permits unrestricted use, distribution, and reproduction in any medium, provided the original author and source are credited. 
2. The second neurogenetic phase- involves the proper functioning of genes that are involved in the conscious experience and run in tandem with neural correlates of consciousness at a different sub-structural scale, i.e., beneath the level of the neuron.

3. The third neurogenetic phase- involves genes that become less active later in life that contribute to mild cognitive impairment and dementia; and gene mutations that contribute to neurodegenerative diseases that affect the degree of human consciousness and interface with the environment by causing defects, death, or gross loss of the neurons in the brain. As far as the study of gene mutations that are correlated with neurodegenerative diseases and their effect on human consciousness, Alzheimer disease (AD) serves as prefect a research model [4].

This neurogenetic model will allow investigators to objectively view human consciousness neurogenetically, not only from a philosophical context [5] but also from an objective neurogenetic context as well [6]. Moreover, we can see what neurodegenerative diseases, e.g., $\mathrm{AD}$ can tell us about the neurogenetic link to human consciousness [4]. For example, gene mutations that induce neuron loss later in life can have a direct effect on certain modalities of the consciousness complexity and interaction. However, new gene-based therapies that have been summarized in previous work [7] seem to indicate that diseases like $\mathrm{AD}$ may one day be reversed. This suggests that the cure in many ways is enhancement, i.e., from the degree of consciousness seen in the pathological state induced by neurodegeneration to what would be considered the subjective median of functioning [8]. In addition, these gene-derived treatments may be used in the future in individuals without neurodegenerative diseases in an attempt to enhance brain performance and human consciousness [7].

Electroencephalographic-derived indexing of human consciousness: In a groundbreaking article by Dr. Adenauer G. Casali et al. "A Theoretically Based Index of Consciousness Independent of Sensory Processing and Behavior", a new tool that uses a mathematicallyderived formula from signals that are recorded from a high-density electroencephalography (HD-EEG) [9]. These signals are obtained while the human subject is undergoing transcranial magnetic stimulation (TMS). This method allows investigators to develop an index that can distinguish between different levels of consciousness in the human brain.

This technique has allowed investigators to detect the presence or absence of consciousness in different states, e.g., in healthy awake individuals (baseline), those whom are sleeping, patients with lockedin syndrome, patients in minimally conscious state (MCS), patients under various types of anesthesia, and patients in a persistent vegetative state (VS) $[9,10]$. In addition, this technique may also have applications in assessing and monitoring individuals with traumatic brain injury and has been projected to have clinical utility in intensive care units to establish levels of consciousness. But how does it work?

TMS is used to activate brain circuits by perturbing the cortex to engage distributed interactions in the brain, i.e., integration. Then these interactions are calculated by compressing the spatiotemporal patterns of the electrocortical responses in order to measure the algorithmic complexity, i.e., information. With this, a complexity measure can be objectively obtained, which is called the perturbational complexity index (PCI). The PCI value; which ranges from 0 to 1 , enables investigators to analyze how the human brain responds to stimulus from the external environment and can in turn reliably discriminate the level of consciousness in a single individual using this simple noninvasive measurement.
TMS-EEG demonstrates promise in identifying electrocortical markers of consciousness in the human brain and affords the opportunity to give an objective method to measure human consciousness. Tying in the neurogenetic model, it is interesting to point out that the DNA is still active (or some genes could be inactive) in the unconscious state and in disorders of consciousness, but this area of science is poorly understood.

Limbic regions and human consciousness: The default mode network (DMN) is the baseline activity when the brain is idle, i.e., awake but with the individual's eyes closed; and comprises groups of neurons found in the anterior and posterior midline cingulate that connects the parietal and hippocampal regions. These regions become less active when the brain pays attention to something in the external environment [11]. In patients with MCS and VS there is a widespread cerebral and DMN hypoconnectivity.

A recent study, by Dr. Carol Di Perri et al. "Limbic Hyperconnectivity in the Vegetative State", it was demonstrated that there is a paradoxical hyperconnectivity in limbic structures- specifically in the orbitofrontal cortex, insula, hypothalamus, and ventral tegmental area, in patients with MCS and in VS [12]. These results were obtained using restingstate MRI temporal series. The findings suggest that the persistent engagement, i.e., hyperconnectivity of the limbic system- perhaps by self-reinforcing neural loops, may be responsible for the disruption in the normal patterns of connectivity in the human brain. Moreover, this increase in limbic system connectivity may be responsible for the decrease in human consciousness seen in disorders of consciousness. Hence, further study on limbic hyperconnectivity may shed light on the fine line of demarcation between the states of human consciousness and unconsciousness.

\section{Summary}

In this paper we have seen two novel paradigms that change how human consciousness is defined. Dr. Close and Dr. Neppe have made the conceptualization more precise in terms of what type or part of consciousness is being defined and furthermore, how it is compartmentalized. In the neurogenetic model it is elucidated how $\mathrm{NgCC}$ are involved in human consciousness from the development of the brain, during the lifespan, and during neurodegeneration- in which $\mathrm{AD}$ can be used as a pathological example. In addition, the neurogenetic model proposes that neurodegeneration may someday be reversed with gene-derived therapies. However, the neurogenetic model also confronts the reality that these same therapies may one day be utilized to enhance human consciousness in humans without a neurological disorder.

Additionally, two significant discoveries have been summarized that have changed the manner in which we objectify human consciousness neurophysiologically. Dr. Casali et al. and Dr. Gosseries et al. have developed an understanding of an index, the PCI, which objectifies the human brain electrophysiologically in terms of interactions and the complexity of response in quantifiable expressions. Moreover, Dr. Perri et al. has demonstrated that there is still more to learn in our understanding of the human brain neurophysiologically. This was highlighted with new findings in parts of the limbic system in patients with MCS and VS. This further illustrates that our understanding of the human brain is still incomplete, in so far as to what specific brain regions are doing during consciousness, unconsciousness, and in various disorders of consciousness.

\section{References}

1. Neppe, Vernon M, Close ER (2014) EPIC Consciousness: A Pertinent New 
Citation: Grandy JK (2015) New Methods for Defining and Investigating Human Consciousness: 2015. J Neurol Disord 3: e114. doi: 10.4172/23296895.1000 e114

Page 3 of 3

Unification of an Important Concept. Journal of Psychology and Clinical Psychiatry 1: 1-36.

2. John G (2013) The Neurogenetic Correlates of Consciousness. In: Amoroso RL, Kauffman LH, Rowlands P: The Physics of Reality: Space, Time, Matter Cosmos, 8th Symposium in honor of Jean-Pierre Vigier Singapore: World Scientific, Singapore.

3. John G (2013) The Three Neurogenetic Phases of Human Consciousness. The Journal of Conscious Evolution 9: 1-24.

4. John G. Alzheimer Disease and Human Consciousness: A Neurogenetic Connection.

5. John G (2014) The Neurogenetic Substructures of Human Consciousness. Essays in Philosophy 15: 266-278.

6. John G (2014) Neurogenetics and Human Consciousness. The Journal of Neurological Disorders 2: 1-3

7. John G (2014) The Three Neurogenetic Phases of Human Consciousness: The
Possibility of Transhuman and Posthuman Consciousness. The International Journal of Arts and Sciences 7: 381-394.

8. John G (2010) Selected Genetic Destination: The Rise of Homo sapiens genomicus. International Journal of Arts and Sciences; 3: 166-190.

9. Casali AG, Gosseries O, Rosanova M, Boly M, Sarasso S, et al. (2013) A Theoretically Based Index of Consciousness Independent of Sensory Processing and Behavior. Science Transitional Medicine 5: 105

10. Olivia G, Thibaut A, Boly M (2014) Assessing Consciousness in Coma and Related States Using Transcranial Magnetic Stimulation Combined with Electroecphalography. Ann Fr Anesth Reanim

11. Vanhaudenhuyse A, Noirhomme Q, Tshibanda LJ, Bruno MA, Boveroux P, et al (2010) Default network connectivity reflects the level of consciousness in noncommunicative brain-damaged patients.Brain 133: 161-171.

12. Di Perri C, Bastianello S, Bartsch AJ, Pistarini C, Maggioni G, et al. (2013) Limbic hyperconnectivity in the vegetative state.Neurology 81: 1417-1424. 